\title{
Origin and early evolution of photosynthesis: a brief historical account
}

\author{
Tanai Cardona \\ Department of Life Sciences, Imperial College London, London, UK \\ Correspondence to: t.cardona@imperial.ac.uk
}

ORCID: 0000-0003-2076-4115

Keywords: photosynthesis, photosystem, water oxidation, oxygenic, evolution, origin of life

\begin{abstract}
What if oxygenic photosynthesis is a primordial process with roots at the origin of life? What would the impact of this change in perspective be on our understanding of the early Earth and of the emergence and diversification of life? In here, I will examine some of the historical context of the study of the evolution of photosynthesis, which led to the consolidation of the current notion that the origin of anoxygenic photosynthesis occurred before the origin of oxygenic photosynthesis. I will show with a few examples how the mainstream view on the evolution of photosynthesis traces back to Oparin's 'primordial soup' scenario for the origin of life, fuelled by the century-old perception that oxygenic photosynthesis is a plant rather than a bacterial trait. However, it has become more evident than ever before that the mainstream view is not supported by the evolution of the photosystems. In other words, the origin of biological water oxidation appears to be the seed from where photosynthesis sprout. Somewhat troubling and contrary to all predictions that derive from the mainstream view, photosystem II - the water-splitting and oxygen-evolving enzyme - shows features that are better explained if photochemical reaction centres originated during the establishment of oxygenic photosynthesis. An urgent revision of the evolution of photosynthesis procured to be free from biases of interpretations and presuppositions is strongly encouraged from all angles of the Life and Earth Sciences.
\end{abstract}

\section{Early historical influences}

It is currently accepted that photosynthesis was first anoxygenic and that this originated in a type of bacteria. It is accepted that at some point after the origin of anoxygenic photosynthesis, oxygenic photosynthesis arose in [an ancestor of] cyanobacteria. It is thought that the span of time between the origin of anoxygenic and oxygenic photosynthesis could 
have been several hundred million years, if not a billion years. I will refer to this accepted framework as the mainstream view. Up until now, many scenarios and schemes explaining the evolution of photosynthesis have been discussed in the literature, and some of them might appear contradictory, yet most can be placed within this framework of thought. However, how this mainstream view became consolidated cannot be truly understood without an awareness of the history of the subject (Fig. 1). For a balanced introduction to the evolution of photosynthesis, please refer to Hohmann-Marriott and Blankenship (2011).

Let us begin with one of the most influential works on the evolution of photosynthesis to date, the hypothesis paper by John M. Olson published in 1970 in Science as a tribute to Cornelis B. van Niel (Olson 1970). This paper is of remarkable importance because it described for the first time the evolution of photosynthesis in terms of photosystem evolution (Fig. 1). Olson wrote:

"The evolutionary pressure for the utilization of ever weaker electron donors gradually forced one reaction center toward ever higher redox potentials in order to be able to extract electrons from the new donors. In this manner system 2 evolved from system 1 in a long series of small mutational steps."

A

Influencers

Oparin, van Niel, others 30 s to 70 s

Olson 1970

Today's mainstream view: Pierson and Olson 1980s, via Blankenship 1992

B

Life LUCA Bacteria Anoxygenic $\begin{gathered}\text { Oxygenic } \\ \text { photosynthesis }\end{gathered}$

Figure 1. Chains of influence in the study of the molecular evolution of photosynthesis. A Many proposals for the origin and evolution of photosynthesis have been presented in the literature dating back to the first half of the twentieth century. No other author has had the influence of Oparin and van Niel. It should be noted that 
van Niel himself was a famous, sought after and acclaimed teacher (Barker and Hungate 1990) and his lectures likely influenced thinkers far beyond what can be appreciated by a read of the available literature. This can be clearly appreciated in van Niel (1956), a surviving and accessible record of some of his lectures. Oparin and van Niel's influence on Olson's proposed scenarios can also be appreciated in Olson (1970) hypothesis paper, which became one of the most influential texts in the study of the evolution of photosynthesis. Olson, together with Beverly K. Pierson, then added more detail to their scenarios through the 1980s. Pierson and Olson's work gathered further influence when in conversation with Blankenship's perspective on evolution, as presented in Blankenship (1992) and subsequent jointly authored publications. Is this conversation what characterizes the current perception of our understanding of the evolution of photosynthesis, which today takes the shape of the sequence of events shown in B. Namely, oxygenic photosynthesis is considered to be a late innovation relative to the origin of life and to emanate from a speculative anoxygenic transitional stage $(\mathrm{RC} 1+\mathrm{RC} 2)$, the details of which are usually debated through a Pierson-Olson-Blankenship framework of thought. RC1 denotes ancestral type I reaction centres, while RC2 denotes the ancestral anoxygenic type II reaction centres, the latter conceptually understood as being similar to the purple bacteria reaction centre. PSII and PSI denotes the type II and type I reaction centres used in oxygenic photosynthesis, respectively.

It should be noted here that "system 2" means photosystem II, the water-splitting enzyme of oxygenic photosynthesis. At this point in time, it was not yet understood that "the purple bacteria" had a "quinone-type" or "type II" reaction centre homologous to photosystem II. Therefore, it seemed reasonable to Olson that bacterial "system 1" should have given rise to the more sophisticated "system 2" of algae and plants. Another key point in this statement is the accessibility of electron donors as an evolutionary driving force for the origin of water oxidation. This rationale derives directly from what was known as "the heterotrophic hypothesis", one of the three main explicit assumptions behind Olson's evolutionary speculation. The heterotrophic hypothesis refers to Oparin's origin of life scenario in a primordial soup rich with organic compounds, which was then the most popular perspective providing context for the origin of photosynthesis. This is described more eloquently in the 
hugely influential and elegant voice of van Niel. The following text is part of a series of lectures compiled in a book published in 1956 (van Niel 1956):

"The first phase of biochemical evolution would therefore have involved the gradual acquisition of the ability to use progressively less complex mixtures of organic ingredients for the production of the chemical constituents of the organisms. This phase can thus be characterized as an evolution toward increased independence on a medium replete with a multitude of organic materials. The theoretical limit of such independence is reached with the appearance of organisms that no longer require any organic substances. These are the chemo- and photoautotrophic organisms. [...]"

\section{"[...] While the chemoautotrophs are no longer dependent on a supply of} organic substances, the photoautotrophs have thus acquired a new metabolic feature, which in the end makes them independent even of oxidizable inorganic matter, still needed by the chemoautotrophs for the assimilation of carbon dioxide. But the photosynthetic bacteria, too, require oxidizable inorganic substances, such as hydrogen sulfide or molecular hydrogen. Metabolically they are therefore intermediate between the chemoautotrophs and the green plants."

Thus, the scenario proposed by Olson, which introduced to a broad readership the very first photosystem evolutionary sequence, are directly derived from Oparin's and van Niel's confident views on evolution. Olson using this framework imagined then a series of candidate electron donors to "system 2" that would have been used as intermediate transitions before water, including hydrazine and hydroxylamine.

Several other important aspects on van Niel's text above should be noted here. Firstly, even though cyanobacteria had been known to be bacteria at this point, at a conceptual level they had never stopped being "green plants". Therefore, there has always been a bias in perception of cyanobacteria, and in consequence of oxygenic photosynthesis, as being less primitive than other photosynthetic bacteria, simply because historically they have been affiliated to the realm of plants. The effects of this historical legacy are still felt today. More notably, in the issues that have arisen with the modernization of bacterial classification (Oren and Ventura 2017, Oren 2020). Yet still today within the photosynthesis research community, 
bacterial photosynthesis and bacterial reaction centres still mean anoxygenic photosynthesis and its reaction centres.

Secondly, it was not until the late 70s that the 'domains of life', Archaea and Bacteria, started to become formalized (Fox et al. 1980) and therefore discussions on whether a metabolic trait can be traced back to before or after the "last universal common ancestor" were not conceptually possible. Thus, Olson's and other less impactful perspectives on the origin of photosynthesis before the 90s, inevitably see the emergence of the trait as a natural progression from a primordial soup origin. Nevertheless, in the $80 \mathrm{~s}$, perspectives on the evolution of photosynthesis derived from Oparin's heterotrophic hypothesis had been firmly internalized for decades, so that they were presented as fact, even in the absence of any data supporting these. A nice picture of this situation was fortunately captured in the Proceedings of the First International Symposium on the Origin of Life on The Earth, held in Moscow in 1957. Perspectives on the evolution of photosynthesis well aligned with Oparin's way of thinking permeate many of the contributions (Oparin et al. 1959). See contributions by Bernal, Calvin, or Krasnovsky, for example, among several others. The interpretative problem arising from Oparin's and van Niel's influence regarding the origin and evolution of photosynthesis was sharply criticized by Carl R. Woese (Woese et al. 1985, Woese 1987), as he presented data consistent with a photosynthetic origin of bacteria. Woese and colleagues noted in their 1985 paper:

\section{"[...] Although we still have no idea whether such an idea is correct, it tends} to be presented to each new generation of microbiologists as the unassailable truth."

Their warning had no impact — at least with regards to the understanding of the evolution of photosynthesis. However, subsequent work on the tree of life did eventually lead to the notion that photosynthesis originated in a group of bacteria. In consequence, today it is usually thought that the domain Bacteria was unlikely to be ancestrally photosynthetic; and it is thought even less likely that photosynthesis had roots at the origin of life. The warning is still relevant, nonetheless. For example, when I attempted to reassess the evolution of photosynthesis for the first time (Cardona 2015), trying somewhat unsuccessfully to provide a fresh and impartial reassessment of available data — not so difficult since I was not as well read-I did end up concluding that photosynthesis was likely an ancestral trait of bacteria. 
It was not until the late 70s and early 80 s that the similarities of the anoxygenic type II reaction centre from purple bacteria and photosystem II led to the first suggestions that these two might be homologous (Olson 1981). These were based entirely on biochemical and biophysical characterizations, but in the absence of sequence or phylogenetic data. See also Blankenship and Parson (1978) who reviewed and noted the many similarities between "bacterial reaction centers" and "plant photosystem II", although evolution of the systems was not explicitly considered. By then, it was already too late for the evolutionary implications of this emerging data to be interpreted objectively, because at the time it was believed without place for doubt, that anoxygenic photosynthesis was more primitive than oxygenic. This unavoidably translated into the notion that the anoxygenic type II reaction centre of the purple bacteria was indeed more primitive than the oxygen-evolving photosystem II, and that the former must have given rise to the latter. Thus photosystem II soon became "a somewhat abnormal purple bacteria reaction centre", to put it in the exact words of A. W. Rutherford (1989). Hence, it has always been implicitly accepted as fact that the anoxygenic type II reaction centre was ancestral to photosystem II. This view is unambiguously incorrect (Fig. 2 and Fig. 3). 


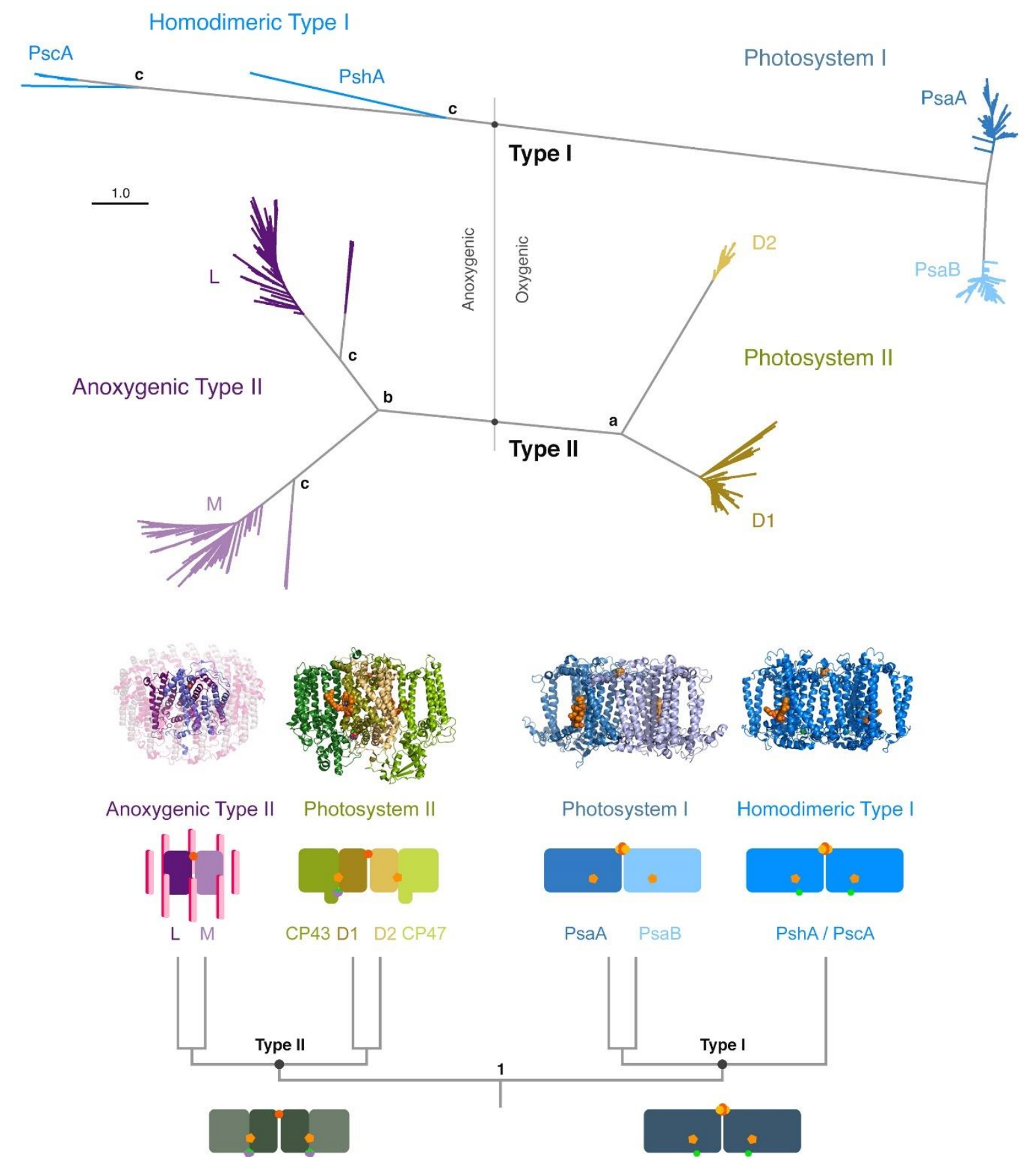

Figure 2. Evolution of photosynthesis. A Phylogenetic relationships between reaction centre proteins. B the same as A but including additional structural information. The early history of photosynthesis is clearly recorded in the phylogeny of the reaction centre proteins and this can be interpreted unambiguously because of several well constrained transitions. Firstly, all reaction centre proteins have a common origin (marked $\mathbf{1}$ in B). L, M, D1, and D2, the type II reaction centre proteins, make a monophyletic clade. PsaA, PsaB, PshA, and PscA, the type I reaction centre proteins, make a distinct monophyletic clade. Therefore, the earliest 
diversification event in the evolution of reaction centre proteins is the divergence of type II and type I. We can deduce this unambiguously because the type I reaction centre proteins share among themselves greater sequence identity, and retain much greater functional and structural similarities, than when compared with type II reaction centre proteins, and vice versa. This means that the divergence of type I and II reaction centre proteins must antedate any other diversification event that follows. In other words, it must antedate the diversification events leading to the phylum-specific reaction centre proteins. Because the relationship between type I and II reaction centres is unambiguous, it can be concluded unequivocally that the evolutionary events leading to type I and II occurred, at the very least, near the root of the tree of life of Bacteria, but likely before that when other relationships are taken into consideration, as described next, but see also Oliver et al. (2021). The large amount of sequence change (i.e. evolutionary distance, grey branches in A) that separates the different reaction centre proteins represent gaps in diversity that can only be explained by loss and extinction events. More likely loss of photosynthesis because these distances are not empty, they are indeed occupied by a large amount of diversity. While horizontal gene transfer can explain a misplacement of branches like Gemmatimonadetes sequences found within Protoebacteria (not shown, but see Zeng et al. (2014)), it cannot explain the distance that separates PshA from PscA, or the distance that separate D1 from D2. Secondly, after the type I and II divergence, a deep dichotomy is seen separating the lineage of reaction centre proteins that are today used in oxygenic and anoxygenic photosynthesis (vertical grey line in A). This pattern is unambiguously constrained in type II reaction centres by the independent duplication events leading to D1 and D2 (a), and to L and M (b), that are clearly observed at a sequence and structural level too. In type I reaction centres it is unambiguously constrained by the observation that PshA and PscA share much greater sequence identity and structural conservation than when compared with photosystem I's PsaA and PsaB subunits. Therefore, and contrary to the mainstream view, the evolution of the photosystem does not support the notion that anoxygenic photosynthesis gave rise to oxygenic photosynthesis, and unambiguously shows that the photosystems today found in Cyanobacteria and plastids are, at the very least, as old as those found in anoxygenic photosynthesis. It appears however that the diversification events leading to the distinct "oxygenic" and "anoxygenic" lineages, including the D1/D2 (a) and L/M (b) duplications are still so ancient that they occurred before bacteria started to radiate; in such a way that only the divergence of phylum-specific anoxygenic type II reaction centres and homodimeric type I reaction centres should be 
considered coincidental with the diversification of known bacterial groups (c). Nonetheless, at a functional and structural level it can be concluded that the ancestral type II reaction centre, before L/M/D1/D2 was architecturally and functionally more like photosystem II, as described in the main text and indicated references, and in Fig. 3. This suggests that the ancestors to L/M, D1/D2, PshA/PscA, and PsaA/PsaB may have been paralogues of reaction centre subunits during the early establishment of oxygenic photosynthesis, co-occurring with each other in the same genome: a trait that has only evolved in the context of oxygenic photosynthesis, and, as it still occurs in Cyanobacteria today (Oliver et al. 2021)

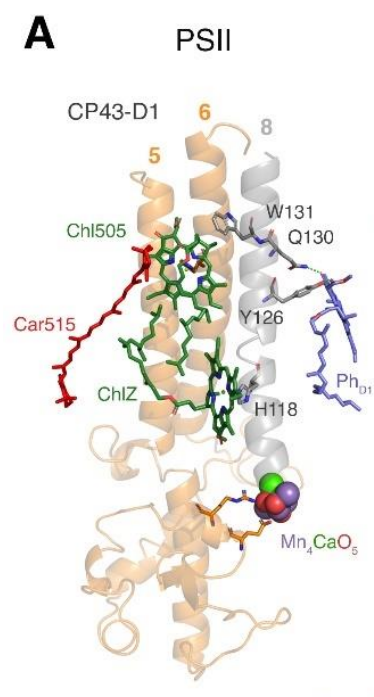

\section{B PSII/HbRC}

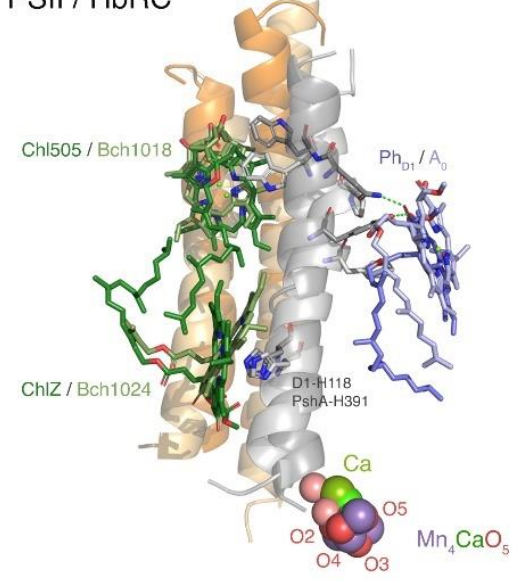

HbRC

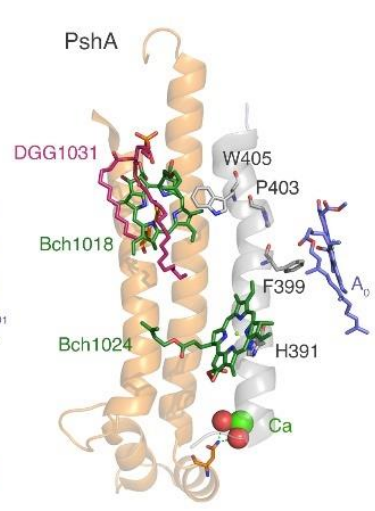

C

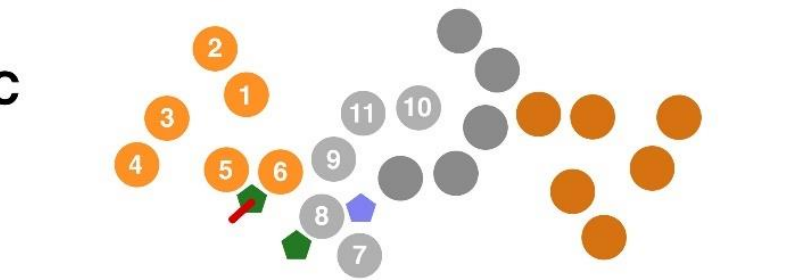

D

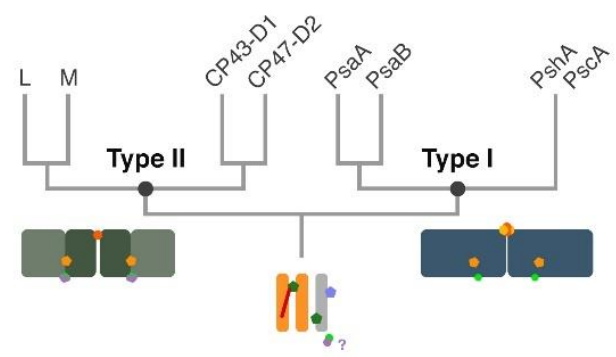

Figure 3. The earliest type II reaction centre was in many ways like water-splitting photosystem II. A and $\mathbf{B}$ show structural visualizations of the region around the $5^{\text {th }}$ and $6^{\text {th }}$ transmembrane helices of the antenna domain (orange ribbons), and the $8^{\text {th }}$ helix of the reaction centre core domain (grey ribbons). PSII denotes photosystem II (Cyanobacteria and plastids); HbRC, the heliobacterial reaction centre (Firmicutes); GsbRC, the green sulfur bacterial reaction centre (Chlorobia); PSI, photosystem I (Cyanobacteria and plastids). B shows an overlap of the same region in photosystem II and the HbRC. C shows a schematic 
top-view representation of the position of the transmembrane helices and pigments shown in A and B. Pigments found at homologous positions are shown in similar colours. Antenna chlorophylls (Chl) and bacteriochlorophylls (Bch) are shown in green. Note that ChlZ-H118 (PSII), Bch1024-H391 (HbRC), Bch814-H487, (GsbRC), and Chl1136-H540 (PSI) are homologous, but are bound by the reaction centre core. $\mathrm{Ph}_{\mathrm{D} 1}$ denotes the pheophytin molecule characteristic of type II reaction centres, and $\mathrm{A}_{0}$, the primary acceptor of type I reaction centres, all of which are chlorophyll $a$-derived pigments (light blue). The overlap of these region in PSII and the HbRC highlights the nearly identical positions of the pigments, including the folding of their hydrophobic tails and the $\mathrm{Ca}$-site of the $\mathrm{HbRC}$ relative to the $\mathrm{Ca}$ in the water-oxidizing cluster of PSII. The overlap of the Ca-site and the water-oxidizing cluster shows that the oxygen atom of a water molecule bound to the $\mathrm{Ca}$ in the $\mathrm{HbRC}$ overlaps with an oxygen atom in the water-oxidizing cluster known as 'O2', following the nomenclature of Umena et al. (2011). Both $\mathrm{O} 2$ in the water-oxidizing cluster and the overlapping water molecule in the $\mathrm{Ca}$-site of the $\mathrm{HbRC}$ are hydrogen-bonded by a residue found within the extrinsic loop between the $5^{\text {th }}$ and $6^{\text {th }}$ transmembrane helices. The second water bound to the $\mathrm{Ca}$ in the $\mathrm{HbRC}$ also overlaps with a water molecule bound to the $\mathrm{Ca}$ in the water-oxidizing cluster known as 'W4' (not shown for clarity). Other striking similarities are described in Cardona and Rutherford (2019) and see also Gisriel et al. (2021). D1-Y126 (PSII) and PshA-F339 (HbRC) occupy homologous position, but Y126 provides a hydrogenbond to $\mathrm{Ph}_{\mathrm{D} 1}$. Q130, which in PSII also provides a hydrogen-bond to $\mathrm{Ph}_{\mathrm{D} 1}$, is replaced by proline in the HbRC, likely an insertion relative to D1-Q130. This proline creates a kink in the $8^{\text {th }}$ helix inducing a rotational change of PshA-W405 that is in van der Walls contact with Bch1018, relative to D1-W131 in contact with conserved pigment Ch1505 in the CP43 subunit. What appears to be a conserved carotenoid interacting with Chl505 in PSII (red pigment), is also found in the GsbRC and in PSI. In PSI, the equivalent chlorophyll to Car505 has been displaced in PsaA by a second carotenoid. This feature of PsaA is also conserved in PsaB (not shown). In the HbRC the interaction with an equivalent carotenoid is replaced by an interaction with a lipid. It suggests that this position has been under pressure to enhance photoprotection from excess excitation from early on (Gisriel et al. 2021). D These structural relationships show that the "architecture of photosystem II" is not only ancestral to type II reaction centres, but it is also required and optimized to catalyse and sustain water oxidation. In consequence, photosystem II cannot be explained as a purple bacteria reaction centre that just simply "acquired" an antenna domain and a Mn cluster. It means that the divergence 
leading to type II and type I reaction centres happened within the evolution of oxygenic photosynthesis, and the evolutionary pressures that this imposed in the nascent reaction centres: both at the level of the optimization of an electron transfer chain from water to metabolism, and at the level of protection against photodamage.

\section{A modern interpretation}

In 1990, the first detailed phylogenetic analysis of type II reaction centre protein sequences was presented by Timothy J. Beanland. Beanland (1990) perceptively noted that even though it was clear that the purple bacteria reaction centre and photosystem II were homologous, the evolutionary relationships between these had not been adequately addressed. Beanland noted that the phylogeny of type II reaction centres did not quite align with the expectations of the time. He stated:

"These results suggest that PS-II may be more ancient than previously supposed."

"Hypothesis-testing suggests the scheme shown in Fig. 4 in which PSIIemerges as the most deeply-rooting of the tree "Q-type" RCs. This is in agreement with recent findings that suggest the similarities between the purple bacterial $R C$ and PS-II have been exaggerated, and cautions against over-extrapolation from the former to the latter."

Nevertheless, to re-align his results to the mainstream view, Beanland then minimizes the implications of the findings by suggesting that a greater antiquity for photosystem II than previously supposed should not be taken as meaning that oxygenic photosynthesis antedated anoxygenic photosynthesis, speculating that:

“[...] it is perhaps more likely that a primitive 'PS-II' acted cyclically as seen in purple and green bacterial RCs."

Sometimes the weight of a historical legacy is heavier than the weight of data. Beanland's paper had little to no impact on the evolutionary thinking of the time. A second phylogenetic 
analysis of type II reaction centre proteins was presented in what I consider to be the most seminal paper in the study of the evolution of photosynthesis to date, published in Photosynthesis Research by Robert E. Blankenship (1992). This analysis confirmed Beanland's observations, but instead placed emphasis in the fact that the topology of the tree did not match the $16 \mathrm{~S}$ rRNA phylogenies of the time, suggesting the possibility of horizontal gene transfer. This was also remarked on by Beanland though. While the $16 \mathrm{~S}$ phylogenies were not quite adequately interpreted by neither Beanland nor Blankenship, unlike Olson and Pierson (1987) before them, the most important aspect of the tree of type II reaction centre proteins: the fact that it cannot be concluded that photosystem II evolved after or from the purple bacteria reaction centre, was not considered in Blankenship (1992), which went on to become one of the most highly cited papers on the topic.

In Blankenship (1992) the new emerging perspective on the impact of horizontal gene transfer was contrasted against Pierson and Olson (1989)'s most recent scenario on the evolution of photosynthesis. At that point, their scenario had been substantially elaborated upon with regards to Olson (1970)'s one. It restated that type I reaction centres were primordial and suggested that the divergence of type I and type II reaction centres occurred before the diversification of bacteria, with the scattered distribution of photosynthesis explained by loss. These seemingly contrasting perspectives opened the door to the most enduring debate in the study of the evolution of photosynthesis: did photosynthesis originate deep within bacteria and was loss repeatedly many times across the tree of life? Or did it emerge in a particular group of bacteria and then scatter across the tree of life via horizontal gene transfer? The debate embodies today's mainstream view of the evolution of photosynthesis, and while many scenarios and rationales have been proposed, they all fit within this framework of discussion. As an example of this, two subsequent evolutionary sketches, published over a decade apart, can be highlighted: that by Mulkidjanian et al. (2006) and by Martin et al. (2018), see their Fig. 1 and Fig. 6, respectively. Contextually they would appear as different scenarios, but essentially they are Pierson and Olson (1989)'s, yet replacing loss events with gene transfer events as in Blankenship (1992).

There is really no debate, because as I remarked before (Cardona 2019), all evolutionary scenarios that we have had available, whether favouring loss or horizontal gene transfer, embed assumptions that lack support built upon other assumptions that lack support. For example, the assumption that ancestral type II reaction centres were anoxygenic, is built upon the assumption that "bacterial photosynthesis" is more primitive than "plant-type 
photosynthesis", leading to the unproven assumption that there was an ancestral anoxygenic cyanobacterium with an anoxygenic type II and a type I reaction centre prior to the emergence of water oxidation.

With these historical considerations in mind, how can therefore be taken as fact that anoxygenic photosynthesis gave rise to oxygenic photosynthesis? I fear that one day this idea will be considered the biggest blunder in evolutionary biology.

\section{A new revision: simple, but radical}

It becomes imperative to reassess the evolution of photosynthesis with fresh eyes, with an active awareness of the history and ideas that have influenced our thinking. When we extract ourselves from the historical context of the subject, it becomes immediately clear that the evolution of photosynthesis is not particularly difficult to understand. This is because there are just two types of photosystems, found in just a few lineages of bacteria. Some of the key relationships between several of the reaction centre proteins are also unambiguous, because they are constrained by a small set of unequivocal gene duplication events (Fig. 2) and a set of conspicuous functional and structural specializations (Fig. 2 and 3). For example, the divergence of type I and II reaction centres, or the independent gene duplications leading to L and M, and to D1 and D2 (Fig. 2). Today, we also have accumulated a very precise and detailed atomic understanding of the photosystems, which means that we can read their evolution at a level of atomic detail that could make some readers uncomfortable, as demonstrated in Fig. 3, or just terribly bored, if compared with preceding evolutionary narratives.

The biologist may feel compelled to believe that there must be unequivocal evidence proving that anoxygenic photosynthesis gave rise to oxygenic photosynthesis entirely based on the rock record. This is not the case. Discussing the early geochemical record of photosynthesis is outside the scope of this paper, and my expertise, but I do wish to highlight a recent review by Planavsky et al. (2021) that illustrates how biological perspectives, in not small amount, contribute to shaping thinking on the topic when approached from an Earth Sciences perspective. The authors wrote:

\section{"Phylogenetic analyses almost unanimously show that anoxygenic} photosynthesis evolved prior to oxygenic photosynthesis [Citing Fischer et al. (2015) and Cardona et al. (2019)] (however, see also ref. [(Cardona 2019)]), and, thus, likely 


\section{had exclusive and unfettered access to the photosynthetic niche for hundreds of millions of years prior to the emergence of oxygenic photosynthesis [...].}

Fischer et al. (2015) and Cardona et al. (2019) are both examples of conflicting perspectives that fall within the mainstream view. One could argue that Fischer et al. (2015) aligns with the "horizontal gene transfer" scenario, while Cardona et al. (2019) represents a variation on the "loss of photosynthesis" scenario. I should note however, that at the time of publication of Cardona et al. (2019), we were still assuming that water-splitting photosystem II emerged from an ancestral anoxygenic type II reaction centre as historically assumed based on the mainstream view. In Cardona et al. (2019) we argued that a water-splitting photosystem II could have antedated the last common ancestor of Cyanobacteria by well over a billion years. A notion with implications radical enough that translated into two years of peer-review and seven rejections from five different journals. Only as I attempted to find clearer ways to communicate and justify to peer-reviewers observations that I thought should have been somewhat straightforward, but that become obfuscated by the history of the subject and its core assumptions, was I finally able to realize the inherent flaws in the mainstream view. Ultimately, it led to my first formal critic of the issue in Cardona (2019). The follow-up work to Cardona et al. (2019), Oliver et al. (2021), started freer from the core assumptions.

What has emerged from the studies of photosystem II by myself and my colleagues is that the water-splitting enzyme is a better model for what an ancestral type II reaction centre looked like than the purple bacterial reaction centre (Fig. 3). Structural and functional characteristics that can be traced back to the earliest type II reaction centres, at the dawn of photosynthesis, are better explained in the context of photosynthetic water oxidation. This is evidently seen when the photosystems are compared with each other side-by-side (see Fig. 2B and Fig. 3, for example), and it is more conspicuously noted in the structure of photosystem II by the presence of the core antenna CP43 and CP47 subunits, and the way they interact with the core D1 and D2 subunits. This interaction occurs in a manner that is conserved in type I reaction centres (Fig. 2B and Fig. 3) as we have detailed in previous discussions (Cardona 2019, Cardona and Rutherford 2019, Oliver et al. 2021), but see also (Gisriel et al. 2021).

The evolution of photosystem II suggests that the core duplications leading to D1 and D2 (Cardona et al. 2019), and CP43 and CP47 (Oliver et al. 2021), not only occurred in the context of water oxidation, but that they occurred a very long time before the last common 
ancestor of Cyanobacteria. The big twist upon a twist is that these events can be as old as the oldest enzymes that are placed with greater confidence before the last universal common ancestor, only because of their broader distribution. These timings are strongly constrained by the large evolutionary distances that separate each paralogue, while simultaneously featuring some of the slowest rates of protein evolution of any enzyme known. Rates that are only common in the most conserved enzymes used to reconstruct all-inclusive trees of life. In fact, a case could be made for the core duplications of photosystem II occurring before the duplication leading to the heterohexameric catalytic head of ATP synthase at the dawn of life (Oliver et al. 2021), priming a reconsideration of the origin of bioenergetics.

Given that it has always been thought that the purple bacterial reaction centre was ancestral to photosystem II, researchers have wondered about how the latter obtained its core antenna. Speculations have ranged from horizontal gene transfer (Orf et al. 2018) to the entire reconfiguration of a type I reaction centre (Cardona 2016), just to provide two recent examples. However, there should be no doubt that CP43 and CP47 are the ancestral antenna of type II reaction centres, as they interact with the core (D1 and D2) through structural characteristics conserved in type I reaction centres (Fig. 3). I believe this should have been the initial hypothesis as soon as sequence and structural data of photosystem II and photosystem I started to become available around the turn of the century, but our pursue for answers was misdirected by the history of the subject, including my very own pursuit.

How is the antenna of photosystem II relevant to the study of the evolution of photosynthesis? Why is this important? Because the existence of CP43 and CP47 breaks irreparably the notion that anoxygenic gave rise to oxygenic photosynthesis. The reason photosystem II retains CP43 and CP47, and the reason why they are encoded in separate genes unlike type I reaction centres, is water oxidation. Principally, the CP43 coordinates the water-oxidizing cluster together with D1, and thus water oxidation could not exist in the absence of an antenna domain. While there is no water-oxidizing cluster in CP47-D2, there is evidence that this existed prior to core duplication (Cardona et al. 2019) and the changes leading to the loss of it can be reconstructed from structural comparisons as detailed in Oliver et al. (2021). The major surprise came from the structure of the type I reaction centre of heliobacteria (Gisriel et al. 2017), which revealed a Ca atom bound right at the same position where this is found in the $\mathrm{Mn}_{4} \mathrm{CaO}_{5}$ water-oxidizing cluster of photosystem II (Fig. 3), with a number of structural similarities that would not have been unexpected at all, if not because of the mainstream view (Cardona and Rutherford 2019). 
Secondly, the reason why CP43 and CP47 are separate subunits encoded in different genes to the reaction centre core proteins is also explained in the context of water oxidation, because damage of the core subunits mediated by reactive oxygen species (Vass 2012) leads to different rates of degradation of the core subunits (Yao et al. 2012). Thus, D1 has the fastest turnover rate with half-times under one hour, D2 over three hours, CP43 over six hours, and CP47 over 11 hours in a model cyanobacterium. In contrast, the half-time of various photosystem I core subunits sits between two and three days. The mechanism of degradation and repair also demands a displacement of the CP43 and CP47 subunits for the FtsH complex to access D1 and D2 respectively (Krynicka et al. 2015). Therefore, photosystem II is at an architectural level more similar to type I reaction centres than its anoxygenic cousin, because water oxidation originated before the evolution of purple bacterial reaction centres and because water-splitting catalysis put evolutionary pressures in the system that resulted in the retention of greater ancestral characteristics. It should be noted that this greater similarity between type I reaction centres and photosystem II is only at an architectural level, not at a sequence or phylogenetic level. It follows then that the separation of the antenna and the core in ancestral type II reaction centres occurred because of the pressures for enhanced repair that were demanded to provide tolerance to the production of reactive oxygen species. Contrary to the mainstream view, the loss of antenna domains in the type II reaction centre during the origin of anoxygenic photosynthesis seems to be linked to a move away from oxygenic photosynthesis. This loss was in consequence compensated by the evolution of the novel antenna system characteristic of the purple bacteria reaction centre (Proteobacteria) and the distantly related homologues found in the phylum Chloroflexota (Xin et al. 2018) and Eremiobacterota (Ward et al. 2019).

It can be concluded that the type II reaction centre used in anoxygenic photosynthesis is better described as an abnormal photosystem II. In consequence, we have that one of the earliest events in the history of photosynthesis, the structural and functional specialization that led to two photosystem types, resulted in the establishment of linear electron transfer from water to metabolism. It means that the evolutionary pressures leading to the origin of photosystems, before type I and II, might be better understood if considered within the context of water oxidation catalysis during the earliest history of life.

The question is: what is the impact of this change in perspective on our understanding of the origin of life, the origin of bioenergetics, and the geochemical and ecological transformations of the early Earth? 


\section{Acknowledgements}

I am especially thankful to A. William Rutherford, Thomas Oliver, Patricia Sánchez-

Baracaldo, and Anthony Larkum for their continued support and extensive discussions. The financial support from the Leverhulme Trust (RPG-2017-223) and a UK Research and Innovation Future Leaders Fellowship (MR/T017546/1) is gratefully acknowledged.

\section{References}

Barker, H. A. and R. E. Hungate (1990). Cornelius Bernardus van Niel 1897-1985. Biographical Memoirs of the National Academy of Sciences 59: 1-37.

Beanland, T. J. (1990). Evolutionary relationships between Q-Type photosynthetic reaction centers - Hypothesis-testing using parsimony. Journal of Theoretical Biology 145: 535-545. DOI: $10.1016 / \mathrm{S} 0022-5193(05) 80487-4$.

Blankenship, R. E. (1992). Origin and early evolution of photosynthesis. Photosynthesis Research 33: 91-111.

Blankenship, R. E. and W. W. Parson (1978). The photochemical electron transfer reactions of photosynthetic bacteria and plants. Annual Review of Biochemistry, Vol 83 47: 635-653. DOI: 10.1146/annurev.bi.47.070178.003223.

Cardona, T. (2015). A fresh look at the evolution and diversification of photochemical reaction centers. Photosynthesis Research 126: 111-134. DOI: 10.1007/s11120-014-0065-x.

Cardona, T. (2016). Reconstructing the origin of oxygenic photosynthesis: Do assembly and photoactivation recapitulate evolution? Frontiers in Plant Science 7: 257. DOI: 10.3389/fpls.2016.00257.

Cardona, T. (2019). Thinking twice about the evolution of photosynthesis. Open Biology 9: 180246. DOI: 10.1098/rsob.180246.

Cardona, T. and A. W. Rutherford (2019). Evolution of photochemical reaction centres: more twists? Trends in Plant Science 24: 1008-1021. DOI: 10.1016/j.tplants.2019.06.016.

Cardona, T., P. Sánchez-Baracaldo, A. W. Rutherford and A. W. D. Larkum (2019). Early Archean origin of Photosystem II. Geobiology 17: 127-150. DOI: 10.1111/gbi.12322.

Fischer, W. W., J. Hemp and J. E. Johnson (2015). Manganese and the Evolution of Photosynthesis. Origins of Life and Evolution of Biospheres 45: 351-357. DOI: 10.1007/s11084-015-9442-5.

Fox, G. E., E. Stackebrandt, R. B. Hespell, J. Gibson, J. Maniloff, T. A. Dyer, R. S. Wolfe, W. E. Balch, R. S. Tanner, L. J. Magrum, L. B. Zablen, R. Blakemore, R. Gupta, L. Bonen, B. J. Lewis, D. A. Stahl, K. R. Luehrsen, K. N. Chen and C. R. Woese (1980). The phylogeny of prokaryotes. Science 209: 457-463. DOI: 10.1126/science.6771870. 
Gisriel, C., I. Sarrou, B. Ferlez, J. H. Golbeck, K. E. Redding and R. Fromme (2017). Structure of a symmetric photosynthetic reaction center-photosystem. Science 357: 10211025. DOI: $10.1126 /$ science.aan5611.

Gisriel, C. J., C. Azai and T. Cardona (2021). Recent advances in the structural diversity of reaction centers. Photosynthesis Research 149: 329-343. DOI: 10.1007/s11120-021-00857-9.

Hohmann-Marriott, M. F. and R. E. Blankenship (2011). Evolution of photosynthesis. Annual Review of Plant Biology 62: 515-548. DOI: 10.1146/annurev-arplant-042110-103811.

Krynicka, V., S. Shao, P. J. Nixon and J. Komenda (2015). Accessibility controls selective degradation of Photosystem II subunits by FtsH protease. Nature Plants 1: 15168. DOI: 10.1038/nplants.2015.168.

Martin, W. F., D. A. Bryant and J. T. Beatty (2018). A physiological perspective on the origin and evolution of photosynthesis. FEMS Microbiology Reviews 42: 205-231. DOI: 10.1093/femsre/fux056.

Mulkidjanian, A. Y., E. V. Koonin, K. S. Makarova, S. L. Mekhedov, A. Sorokin, Y. I. Wolf, A. Dufresne, F. Partensky, H. Burd, D. Kaznadzey, R. Haselkorn and M. Y. Galperin (2006). The cyanobacterial genome core and the origin of photosynthesis. Proceedings of the National Academy of Sciences of the United States of America 103: 13126-13131. DOI: 10.1073/pnas.0605709103.

Oliver, T., P. Sánchez-Baracaldo, A. W. Larkum, A. W. Rutherford and T. Cardona (2021). Time-resolved comparative molecular evolution of oxygenic photosynthesis. Biochimica et Biophysica Acta (BBA) - Bioenergetics: 148400. DOI: https://doi.org/10.1016/j.bbabio.2021.148400.

Olson, J. M. (1970). The evolution of photosynthesis. Science 168: 438-446. DOI: 10.1126/science.168.3930.438.

Olson, J. M. (1981). Evolution of photosynthetic reaction centers. Biosystems 14: 89-94. DOI: 10.1016/0303-2647(81)90024-1.

Olson, J. M. and B. K. Pierson (1987). Evolution of Reaction Centers in Photosynthetic Prokaryotes. International Review of Cytology-a Survey of Cell Biology 108: 209-248. DOI: 10.1016/S0074-7696(08)61439-4.

Oparin, A. I., A. E. Braunshtein, A. G. Pasynski and T. E. Pavlovskaya (1959). The Origin of Life on the Earth. London, Pergamon.

Oren, A. (2020). Three alternative proposals to emend the Rules of the International Code of Nomenclature of Prokaryotes to resolve the status of the Cyanobacteria in the prokaryotic nomenclature. Int J Syst Evol Microbiol 70: 4406-4408. DOI: 10.1099/ijsem.0.004268.

Oren, A. and S. Ventura (2017). The current status of cyanobacterial nomenclature under the "prokaryotic" and the "botanical" code. Antonie Van Leeuwenhoek 110: 1257-1269. DOI: 10.1007/s10482-017-0848-0. 
Orf, G. S., C. Gisriel and K. E. Redding (2018). Evolution of photosynthetic reaction centers: insights from the structure of the heliobacterial reaction center. Photosynthesis Research. DOI: $10.1007 / \mathrm{s} 11120-018-0503-2$.

Pierson, B. K. and J. M. Olson (1989). Evolution of photosynthesis in anoxygenic photosynthetic procaryotes. Microbial Mats: Physiological Ecology of Benthic Microbial Communities. Y. Cohen and E. Rosenberg. Washington, American Society of Microbiology: 402-427.

Planavsky, N. J., S. A. Crowe, M. Fakhraee, B. Beaty, C. T. Reinhard, B. J. W. Mills, C. Holstege and K. O. Konhauser (2021). Evolution of the structure and impact of Earth's biosphere. Nature Reviews Earth \& Environment 2: 123-139. DOI: 10.1038/s43017-02000116-w.

Rutherford, A. W. (1989). Photosystem II, the water-splitting enzyme. Trends in Biochemical Sciences 14: 227-232. DOI: Doi 10.1016/0968-0004(89)90032-7.

Umena, Y., K. Kawakami, J. R. Shen and N. Kamiya (2011). Crystal structure of oxygenevolving Photosystem II at a resolution of $1.9 \AA$. Nature 473: 55-60. DOI: 10.1038/nature09913.

van Niel, C. B. (1956). Evolution as viewed by the microbiologist. The Microbe's Contribution to Biology. A. J. Kluyver and C. B. van Niel. Cambridge, Massachusetts, Harvard University Press: 155-176.

Vass, I. (2012). Molecular mechanisms of photodamage in the Photosystem II complex. Biochimica Et Biophysica Acta 1817: 209-217. DOI: 10.1016/j.bbabio.2011.04.014.

Ward, L. M., T. Cardona and H. Holland-Moritz (2019). Evolutionary implications of anoxygenic phototrophy in the bacterial phylum Candidatus Eremiobacterota (WPS-2). Frontiers in microbiology 10: 1658. DOI: 10.3389/fmicb.2019.01658.

Woese, C. R. (1987). Bacterial Evolution. Microbiological Reviews 51: 221-271.

Woese, C. R., B. A. Debrunnervossbrinck, H. Oyaizu, E. Stackebrandt and W. Ludwig (1985). Gram-Positive Bacteria - Possible Photosynthetic Ancestry. Science 229: 762-765.

Xin, Y. Y., Y. Shi, T. X. Niu, Q. Q. Wang, W. Q. Niu, X. J. Huang, W. Ding, L. Yang, R. E. Blankenship, X. L. Xu and F. Sun (2018). Cryo-EM structure of the RC-LH core complex from an early branching photosynthetic prokaryote. Nature Communications 9: 1568. DOI: 10.1038/s41467-018-03881-x.

Yao, D. C., D. C. Brune and W. F. Vermaas (2012). Lifetimes of Photosystem I and II proteins in the cyanobacterium Synechocystis sp. PCC 6803. FEBS Letters 586: 169-173. DOI: 10.1016/j.febslet.2011.12.010.

Zeng, Y. H., F. Y. Feng, H. Medova, J. Dean and M. Koblizek (2014). Functional Type 2 photosynthetic reaction centers found in the rare bacterial phylum Gemmatimonadetes. Proceedings of the National Academy of Sciences of the United States of America 111: 77957800. DOI: 10.1073/pnas.1400295111. 